\title{
An approach to General writer identification problem
}

\author{
Mohammed Tarique ${ }^{1}$, Gufran Malik ${ }^{2}$ \\ ${ }^{l}$ (Department of Mathematics,Dyal singh college College,University of Delhi, India) \\ ${ }_{2}^{2}$ (Department of Mathematics, Ram Lal Anand college (Eve), University of Delhi, India)
}

\begin{abstract}
Writer identification problem has been largely studied in the field of image processing and it has found its application in forensic sciences and other related fields. Previous many works have been done in this area.Most of the problems encountered so far were script specefic inthe sense that they were dealing with the script of a perticulat language at a time e.g Engilsh, Spanish, Hindi etc. In this present work a more generalized problem has been addressed which could deal with the image samples written in diffrent scripts.

Keywords :A.R Coefficient,Classification, Image processing,Pattern Matching, Pattern recognition
\end{abstract}

\section{INTRODUCTION}

"General writer identification problem" is basically is a writer identification problem in which images of handwritings of different authors are there in our database, at this point we are given a sample image of an unknown writer and we have to tell who is the writer of the image sample with the specialty that our data base is not restricted to a particular script and allows different scripts to be there in our database.

Normally in this kind of problem our database consists of single script writing for example Hindi, English, Bengali etc and unknown sample also comes under the same script but in case of General writer identification problem we release this restriction and allow multi-script to be there in our database. Though it seems to be a generalization of single script writer identification problem to multi-script environment but this is not really the case and you yourself will start believing this as we go farther and farther into the detail of discussion.

As far as single script writer identification problem is concerned several excellent work have already been done for script like English, Bengali, Hindi, French etc. For most of the solution, they have used a common way of attacking this problem which is to use pattern recognition technique $i$ e extraction of a set of features from the hand writing of known writer and then based on these features classify the writer of an unknown sample as one of the known writer.

They have mainly concentrated on all-o graph level features on a script under consideration which have been extracted by segmenting the text into lines, words, characters, grapheme s etc. The use of allograph level feature requires knowledge in a particular script ie. how to segment word into character or grapheme etc. And therefore extension of the method based on allograph level features is not straight forward to tackle multi-script problem where writer may write in different scripts. So we need a completely different treatment to solve our problem and need to extract those kind of features which are script independent that's why we need to go through the very low level at the pixel level of the image sample.

Unlike single script writer identification problem not much work have been done in this area so far.

Now in our method the basic idea which we have used is that we have viewed hand writing image as a sequence of pixel value and have tried to predict value of a pixel location by using previous pixel values say last $\mathrm{n}$ terms.

Let for the kth location we want to predict its pixel value say $\mathrm{Y}_{\mathrm{k}}$ by using previous $\mathrm{n}$ terms. Let $\mathrm{Y}_{\mathrm{k}}$ can be written as $\mathrm{Y}_{\mathrm{k}}=\mathrm{a}_{1} \mathrm{Y}_{\mathrm{k}-1}+\mathrm{a}_{2} \mathrm{Y}_{\mathrm{k}-2}+\ldots \ldots \ldots \ldots+\mathrm{a}_{\mathrm{n}} \mathrm{Y}_{\mathrm{k}-\mathrm{n}}+€_{\mathrm{n}}$

Let these a 's, $i=1,2, \ldots ., n$ are such that they are best to predict pixel values of all location then this coefficient vector $\left(\mathrm{a}_{1}, \mathrm{a}_{2}, \ldots . . ., \mathrm{a}\right)$ ) are said to be AR coefficient of this image or image signal. AR coefficient for each image represents that particular writer. Now for the unknown sample it's AR coefficients are calculated 
and it's Euclidean distance with AR coefficients of all other images are calculated and whichever is found to be minimum declared as the hand writing of given image sample.

\section{METHODS}

\section{AR coefficient}

The definition that will be used here is as follows

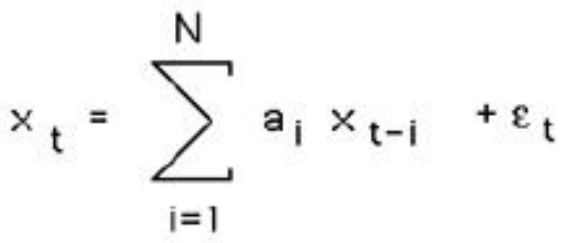

Where $\mathrm{a}_{\mathrm{i}}$ are the auto regression coefficients, $\mathrm{x}_{\mathrm{t}}$ is the series under investigation, and $\mathrm{N}$ is the order (length) of the filter which is generally very much less than the length of the series. The noise term or residue, $\epsilon$ in the above, is almost always assumed to be Gaussian white noise. Verbally, the current term of the series can be estimated by a linear weighted sum of previous terms in the series. The weights are the auto regression coefficients. The problem in AR analysis is to derive the "best" values for a given a series $\mathrm{x}$.

AR coefficients computed from an image written by a specific writer characterizes that writer. Say, there are w writers; each of them contributes one sample. Let $\Theta_{i}$ be the estimated AR model coefficients for the $i$-th writer. For an unknown sample, at first the AR model coefficients are computed. Let $\Theta$ be the estimated coefficients for this sample. Next, the Euclidean distance between this sample and any of the $\mathrm{N}$ samples of the reference database is computed as follows

$$
\mathrm{d}\left(\Theta_{\mathrm{i}, \mathrm{O}} \Theta^{-}\right)=\left\|\left(\Theta_{\mathrm{i}--} \Theta_{0}\right)\right\|^{2}
$$

It is decided that the given sample is written by the $\mathrm{j}$-th writer if $\mathrm{d}(\Theta, \Theta)<\mathrm{d}(\Theta, \Theta)$ for all i.

$\mathrm{j}^{\mathrm{O}} \mathrm{o}(\mathrm{i} \mathrm{o}$

III. Figures AND TABLES

Table-1: Writer identification results on RIMES using different context patterns at different resolutions

\begin{tabular}{|l|l|l|l|l|l|l|l|}
\hline \multirow{2}{*}{$\begin{array}{l}\text { Context Type } \\
\text { (Refer Fig. 1) }\end{array}$} & \multirow{2}{*}{$\begin{array}{c}\text { Image } \\
\text { Resolution }\end{array}$} & \multicolumn{5}{|c|}{$\begin{array}{c}\text { Recognition Results (\% correct) on Rime Dataset } \\
\text { \# Writers: 382, \# Test samples: 100 }\end{array}$} \\
\cline { 3 - 8 } & & Top 1 & Top 2 & Top3 & Top4 & Top 5 & Top 10 \\
\hline $\mathrm{C}_{1}$ & $300 \mathrm{dpi}$ & 55 & 68 & 70 & 70 & 75 & 90 \\
\cline { 2 - 8 } & $150 \mathrm{dpi}$ & 50 & 59 & 62 & 63 & 64 & 72 \\
\hline $\mathrm{C}_{2}$ & $300 \mathrm{dpi}$ & 57 & 66 & 75 & 79 & 79 & 92 \\
\cline { 2 - 8 } & $150 \mathrm{dpi}$ & 47 & 58 & 62 & 64 & 65 & 69 \\
\hline $\mathrm{C}_{3}$ & $300 \mathrm{dpi}$ & 57 & 62 & 70 & 75 & 77 & 97 \\
\cline { 2 - 8 } & $150 \mathrm{dpi}$ & 48 & 55 & 57 & 59 & 68 & 73 \\
\hline
\end{tabular}

\section{CONCLUSION}

The data set which is used to conduct this experiment is RIMES DATA SET . The training set used for writer identification task consists of 300 writers each contributed a letter containing reasonable amount of text. 
The letters are written in French. The test set used here consists of 100 samples. Test samples contain smaller (one-third or less) amount of text than that of the training samples. Each of the writers contributing these test samples has also given training samples. Therefore, ideally there should not be any rejection while identifying the writer for a given test sample. At first, writer identification performance is tested on RIMES dataset. Gray images are converted into binary ones. Results are shown in Table-1. Effectiveness of using three different contexts as shown in fig. 1 are investigated separately. It is observed that bigger contexts outperform smaller one, e.g. 34-order AR coefficients perform better than 24- order coefficients. Identification results in lower resolution are inferior as reported in Table-1 therefore, further experimental results are only reported on the original image resolution, i.e. 300dpi. Interesting to note that top-1 results are not impressive (at best 57\%) but the accuracy rapidly increases to a significant level (at best 97\%) when top 10 choices are considered.

\section{REFERENCES}

\section{Journal Papers:}

[1] R. Plamondon and G. Lorette, "Automatic Signature Verification and Writer Identification - The State of the Art," Pattern Recognition, vol. 22 (2), pp. 107- 131, 1989.

[2] M. Bulacu and L. Schomaker, "Text-Independent Writer Identification and Verification Using Textural and Allographic Features," in IEEE Trans. Pattern- Anal. Mach. Intell. Vol. 29(4), pp. 701-717, 2007.

[3] K. Deguchi, "Two-dimensional Auto-regressive Model for Analysis and Sythesis of Gray-level textures," in Proc. Of the 1st Intll. Sym. for Science on Form, General Ed. S. Ishizaka, Eds. Y. Kato, R. Takaki, and J. Toriwaki, pp. 441-449, Tokyo, Japan, 1986.

[4] ITU-T recommendation T.82, "Information Technology-Coded representation of picture and audio information-Progressive bi-level image compression," March 1993.

[5] E. Grosicki, M. Carré, J. Brodin, and E. Geoffrois, "RIMES evaluation campaign for handwritten mail processing,", in Proc. of ICFHR, 2008.

[6] A. Lemaitre, B.B. Chaudhuri, and B. Coüasnon, "Perceptive Vision for Headline Localisation in Bangla Handwritten Text Recognition," in Proc. of ICDAR, pp. 614-618, 2007.

[7] A. Bensefia, T. Paquet, and L. Heutte, “A Writer Identification and Verification System,” Pattern Recognition Letters, vol. 26 (10), pp. 2080-2092, 2005

[8] R. L. Kashyap, R. Chellappa, and N. Ahuja, "Decision rules for the choice of neighbors in random field models of images," Comput.Graphics Image Proc., vol 